EPJ Web of Conferences 35, 05003 (2012)

DOI: $10.1051 /$ epjconf/20123505003

(c) Owned by the authors, published by EDP Sciences, 2012

\title{
Determination of the angular distribution of evaporation residues following transmission through the superconducting solenoidal separator SOLITAIRE
}

\author{
I.P. Carter ${ }^{1, \mathrm{a}}$, M.L. Brown ${ }^{1, \mathrm{~b}}$, M. Dasgupta ${ }^{1}$, D.J. Hinde ${ }^{1}$, M. Evers ${ }^{1}$, D.H. Luong ${ }^{1}$, A. Wakhle ${ }^{1}$, and E. Williams ${ }^{1}$ \\ Department of Nuclear Physics, RSPE, Australian National University, ACT 0200, Australia
}

\begin{abstract}
A highly efficient superconducting solenoidal fusion product separator has been developed at the Australian National University in order to enable separation and detection of evaporation residues following heavy-ion collisions. The determination of absolute fusion cross-sections requires an accurate knowledge of the transmission efficiency of evaporation residues through the superconducting solenoid, which in turn depends on the angular distribution of evaporation residues exiting the target. Two methods have been developed to extract the angular distributions using the radial distribution and the velocity distribution of the evaporation residues exiting the solenoid. The angular distributions are compared with existing direct measurements of evaporation residue angular distributions.
\end{abstract}

\section{Introduction}

Measurements of fusion cross sections with high precision and accuracy are an essential step in investigating the effects of coherent and dissipative processes on quantum tunnelling in fusion of complex nuclei $[1,2]$. Fusion reactions are usually initiated in the laboratory by energetic ion beams impinging on a target. The beam and target nuclei fuse together to form an excited compound nucleus which de-excites by particle emission, forming evaporation residues (ERs). ERs exiting the target are peaked around a scattering angle of $0^{\circ}$ with respect to the beam axis. The elastically scattered beam particles are also forward peaked, and are at least 4 orders of magnitude more intense. It is thus highly desirable to physically eliminate the beam particles before they reach the ER detectors, which may be achieved through the use of an electrostatic and/or magnetic separator.

A $6.5 \mathrm{~T}$ superconducting SOLenoid for In-beam Transport And Identification of $\underline{\text { Recoiling Evaporation }}$ residues (SOLITAIRE) has been developed at the ANU [3]. It is used to enable spatial separation of the ERs from the intense background of elastically scattered particles. A schematic cross-section view of SOLITAIRE is given in figure 1 . The primary beam is stopped in the axial faraday cup placed behind the target. The elastically scattered particles and ERs enter the magnetic field region filled with low pressure He gas, here they undergo scattering and charge changing collisions with the gas atoms. As a result the trajectories of the ERs closely follow that of the average charge state. The high axial magnetic field of the solenoid deflects both the ERs and the elastically scattered particles, so that they follow helical trajectories that return them back to the beam axis. The elastically scattered particles are stopped by the axial rod and discs. Owing

\footnotetext{
a e-mail: ian.carter@anu.edu.au

b Current address: Navman NZ Ltd., 7-11 Kawana St, Northcote, Auckland, New Zealand
}

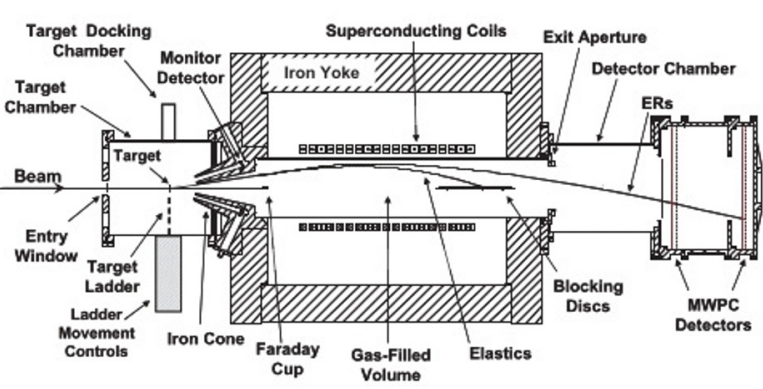

Fig. 1. Schematic cross-section of SOLITAIRE taken from [3].

to their larger magnetic rigidity, the evaporation residues are focused at a point further downstream, which is outside the solenoid. They are then detected in both position sensitive multi-wire proportional counters (MWPCs) beyond this focal position. Along with high precision fusion measurements, SOLITAIRE has the capability to provide low mass radioactive ion beams using the superconducting solenoid as the separator element $[4,5]$.

The large acceptance of the solenoid ( $86 \mathrm{msr}$ ) makes it ideal for measurements of high precision fusion crosssections. The ER transmission efficiency of the separator, whilst large, is less than one hundred percent. The determination of absolute cross-sections using the separator therefore requires an accurate knowledge of the transmission efficiency, which depends on ER angular distributions. The ER angular distribution affect the radial distribution and the velocity distribution of the ERs. Thus, the ER angular distribution can in principle be obtained by two methods:

1. by determining the radial distribution of the ERs measured at the position of two MWPCs (see figure 1).

2. by measuring the velocity distribution of the ERs using the timing information from the two MWPCs.

This paper describes the development of these two methods to enable absolute cross-section determinations using 

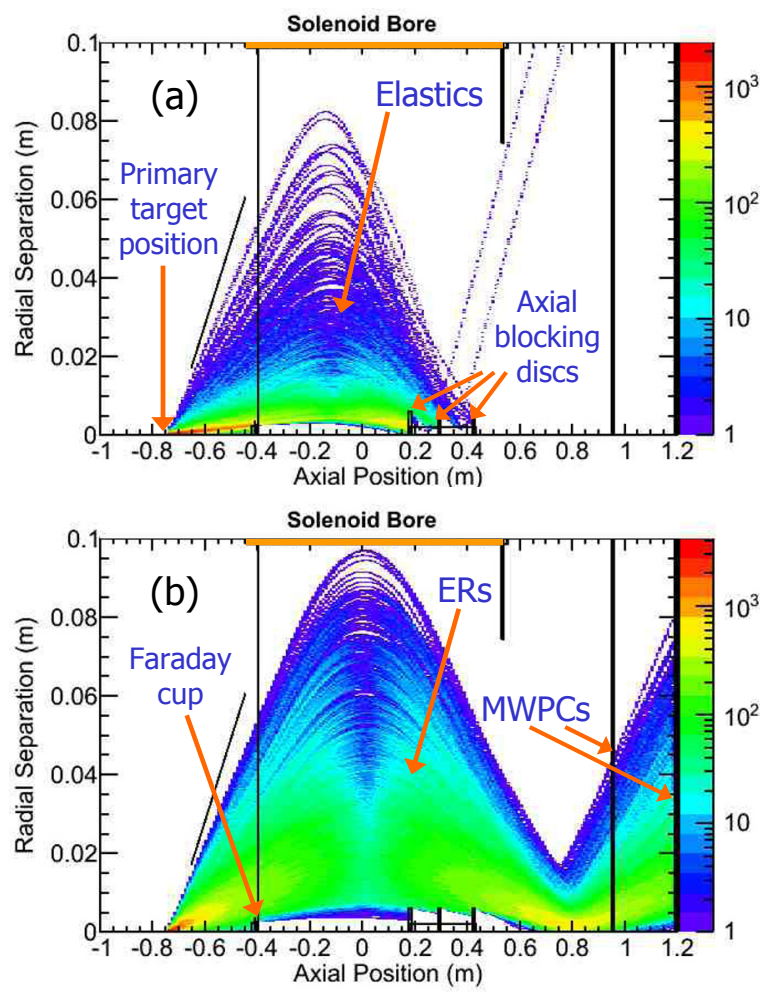

Fig. 2. Ray tracking plots of the trajectories of elastically scattered particles (a) and evaporation residues (b) through the superconducting solenoid. The colour code represents the number of trajectories.

SOLITAIRE. The reliability of the two methods is tested by comparing the results with ER angular distributions measured directly.

\section{Measurement of evaporation residues}

Measurements were made at the Australian National University's Heavy Ion Accelerator Facility. Pulsed beams of ${ }^{34} \mathrm{~S}$ of energy $124 \mathrm{MeV}$ were incident on a ${ }^{89} \mathrm{Y}$ target of thickness $100 \mu \mathrm{g} / \mathrm{cm}^{2}$. The evaporation residues transmitted by the solenoid were detected by two MWPCs, placed at the exit of the solenoid (see figure 1). They each measured the energy loss, position and the time of arrival of the ERs. The X- and y-positions of ERs recored by the MWPC were used to obtain the radial distribution of the ERs at each position of the two MWPCs. The velocity distribution of the ERs were obtained by measuring the time of flight between the two MWPCs.

\section{Radial distribution of evaporation residues}

The trajectories of the ERs and scattered projectiles through SOLITAIRE depends on the magnetic field profile and scattering and charge exchange interactions of the ions with the gas. Incorporating the known geometry of potential obstructions within SOLITAIRE, the probability of ERs emitted at a given angle from the target reaching the MWPC detectors can be calculated.

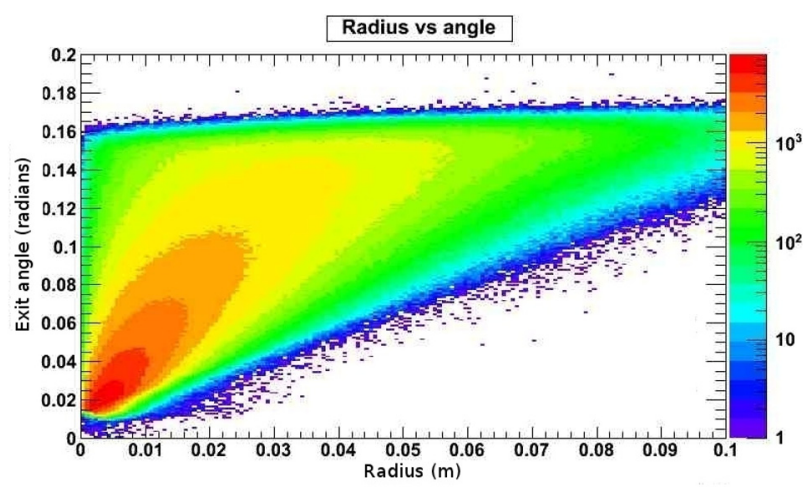

Fig. 3. A typical simulation generated using SOLIRTE showing the relationship between the angle of the ERs exiting the target and their radial position at the MWPC.

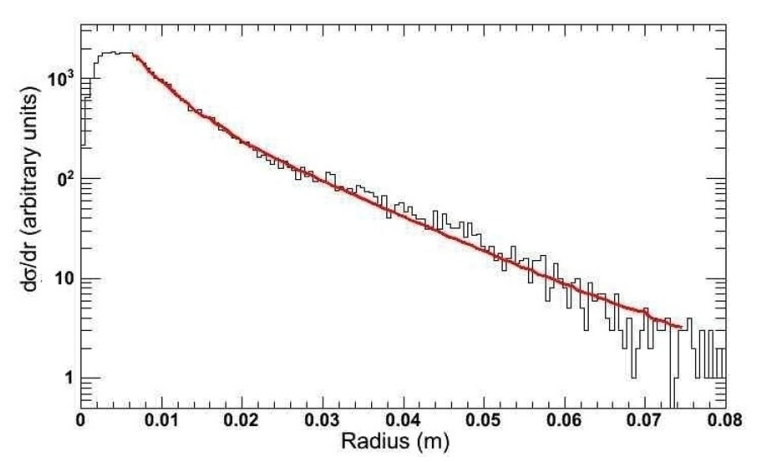

Fig. 4. A typical best-fit ER radial distribution from the simulation (red line) compared with the measurement.

A computer simulation code called SOLIRTE has been developed [6] to calculate the trajectories of the ERs as they traverse the magnetic field and gas filled region. Examples of calculated trajectories of elastically scattered beam particles and ERs are shown in figure 2. To obtain the ER angular distribution from a measurement of the radial position, the first step is to map the relationship between exit angle and radial position by projecting a large number of ER events from the target uniformly over a wide range of scattering angles. The mean charge state in the gas is set to reproduce the experimentally observed focal point. The position of the focus can be determined empirically from the MWPCs by extrapolating the measured trajectory between the two MWPC detectors linearly backwards [7]. The simulations are used to generate a radial distribution for the ER events exiting the target and entering the solenoid within the acceptance angular coverage of $0.45-9.50^{\circ}$. This is used to generate a transmission matrix, an example of which is shown in figure 3. A matrix inversion process is then used to map the radial distribution of the ERs, measured at the position of the MWPCs, to the ER angular distribution from the target. As shown in figure 3, a range of ER angles contributes to each radial position. This mapping enables the determination of the ER angular distribution exciting the target through simulations that best match the measured radial distributions. In order to simulate the ER angular distribution without unphysical fluctuations, it is parameterized in terms of two Gaussian functions centered at $0^{\circ}$ (double Gaussian function). The 


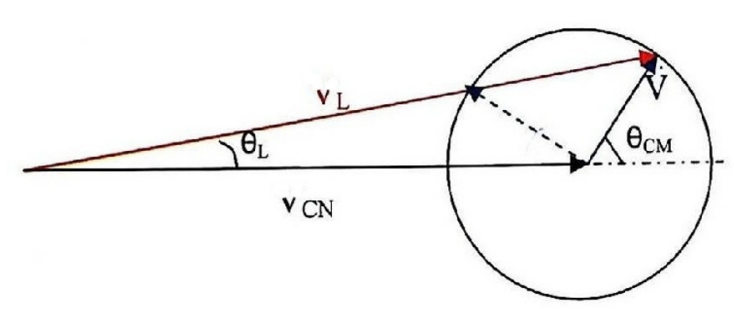

Fig. 5. Diagram indicating the relationship between the angle $\theta_{L}$ and velocity $\mathrm{v}_{L}$ of the ERs in the laboratory frame with the recoil velocity $\mathrm{V}$ of the ERs (resulting from particle evaporation) in the center of mass frame. The ERs with velocity $\mathrm{V}$ in the c.m frame shown by the solid line, and by the dashed line. Both result in ERs appearing at $\theta_{L}$.

parameters are changed in the simulations until a good fit to the measured radial distribution is obtained. The bestfitting Gaussian parameters allow a good reproduction of the measured radial distribution, as shown in figure 4 . The extracted ER angular distribution will be compared in section 5 with those determined from the velocity distribution method described in section 4 below and with direct ER angular distributions measurements.

\section{Velocity Distribution of Evaporation Residues}

ERs are deflected from $0^{\circ}$ by the recoil resulting from the evaporation of particles - mainly neutrons, protons and alpha-particles. In the centre of mass frame, an ensemble of ERs will have a continuous distribution of recoil velocities (V) and angles $\left(\theta_{c m}\right)$. As shown in figure 5 , for a given c.m. velocity vector $\mathrm{V}$, the angle $\theta_{L}$ and velocity $\mathrm{v}_{L}$ of the ER in the laboratory frame are easily calculated, given the initial velocity in the laboratory frame of the compound nucleus $\left(\mathrm{v}_{C N}\right)$. Taking a particular form for the ER angular distribution of $\mathrm{V}$ in the c.m. frame, a Monte Carlo simulation can be used to provide the angular and velocity distributions in the laboratory frame resulting from a single value of $\mathrm{V}$. In this work $\mathrm{V}$ has been approximated by an isotropic distribution. Figure 6 shows Monte Carlo simulations of the velocity distributions (a) and angular distributions (b) for $\mathrm{V} / \mathrm{v}_{C N}=0.035$ and 0.05 .

These distributions can also be easily determined analytically for the case where $\mathrm{V}$ shows an isotropic distribution of $\mathrm{V}$. The algebraic expression which describes the cross section measurements as a function of $\mathrm{v}_{L}$ for a fixed $\mathrm{V}$ is given as

$$
\frac{\mathrm{d} \sigma}{\mathrm{d} v_{L}} \propto\left[\frac{v_{L}}{v_{C N} V}\right]_{v_{L}=v_{C N}-V}^{v_{L}=v_{C N}+V}
$$

The expression which describes the angular distribution for a fixed c.m. recoil velocity $\mathrm{V}$ is

$$
\frac{\mathrm{d} \sigma}{\mathrm{d} \Omega_{L}}\left(\theta_{L}\right) \propto \sum_{i=1,2} \frac{\left(V^{2}+v_{C N}^{2}+2 V v_{C N} \cos \theta_{c m}^{i}\right)^{\frac{3}{2}}}{\left(V^{2}\left(V+v_{C N} \cos \theta_{c m}^{i}\right)\right.} .
$$

Here $\theta_{c m}^{i}$ are the angles of the ERs in the centre of mass frame that result in the ERs appearing at $\theta_{L}$ as shown by the dashed and solid lines in the circle of figure 5. Equations (1) and (2) allowed the development of a relationship between the laboratory ER velocity distribution and the angular distribution via the distribution of $\mathrm{V}$ in the c.m. reference frame. The functions are shown by the full lines in figure 6, and are in good agreement with the Monte Carlo simulations, within the statistical uncertainties of the latter (as indicated by the error bars in the figure).

The ER velocity distribution measured using the two MWPCs for fusion of ${ }^{34} \mathrm{~S}+{ }^{89} \mathrm{Y}$ is shown in figure 7 . It shows narrow and wide components, which can be related to the different recoil velocities associated with nucleon and alpha-particle emission respectively. From inspection of figure 6 and 7, it is apparent that those velocities furthest from the average are associated with c.m. recoil velocities $\mathrm{V}$ which result in the widest components in the angular distribution. Again making use of matrix inversion, a non-zero least squares fitting procedure, with appropriate binning of the $\mathrm{v}_{L}$ distribution, was used to obtain the distribution of $\mathrm{V}$ in the c.m. frame. With the finite statistics in the experimental data, this naturally showed some fluctuations. Although these wil propagate into the angular distribution, as shown in section 5, they are not a significant problem. A reasonable smoothing function applied to the $\mathrm{V}$ distribution can be used to damp these fluctuations.
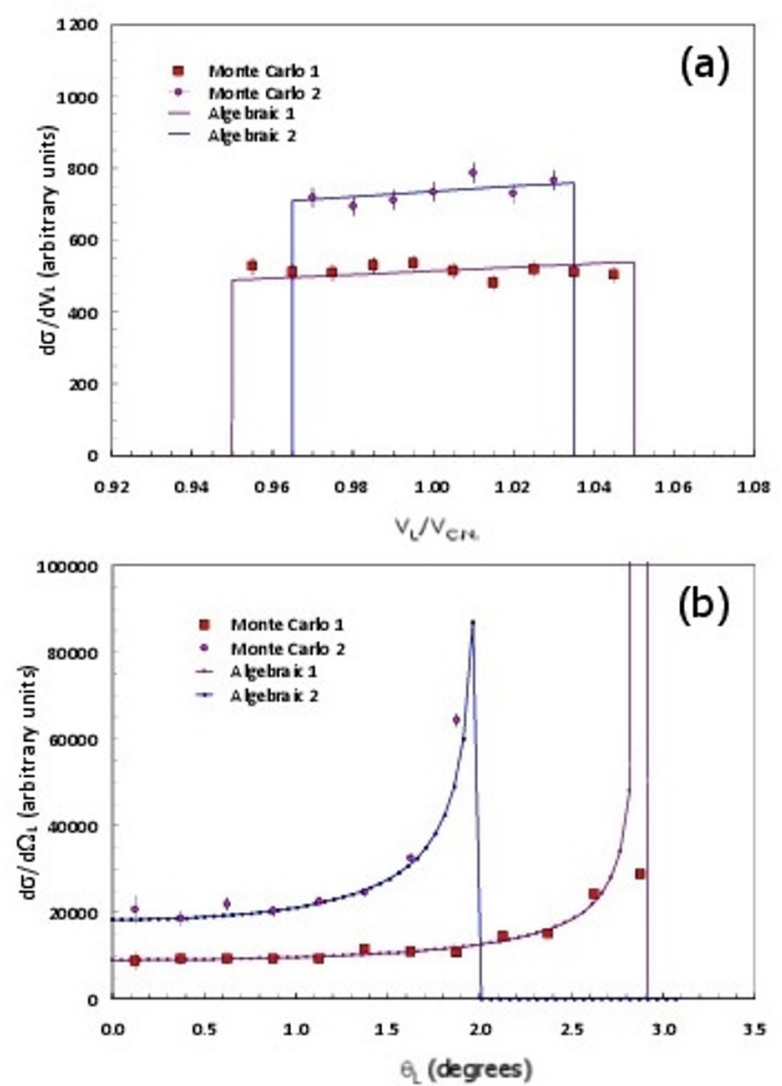

Fig. 6. (a) Differential ER cross-sections for two fixed c.m. recoil velocities corresponding to $5.0 \%$ (case 1) and 3.5\% (case 2) of the compound nucleus velocity $\mathrm{v}_{C N}$ for 5000 events in each case. The points represent Monte Carlo calculations, the lines correspond to algebraic expressions described in the text. (b) The angular distributions corresponding to these fixed c.m. recoil velocities. 


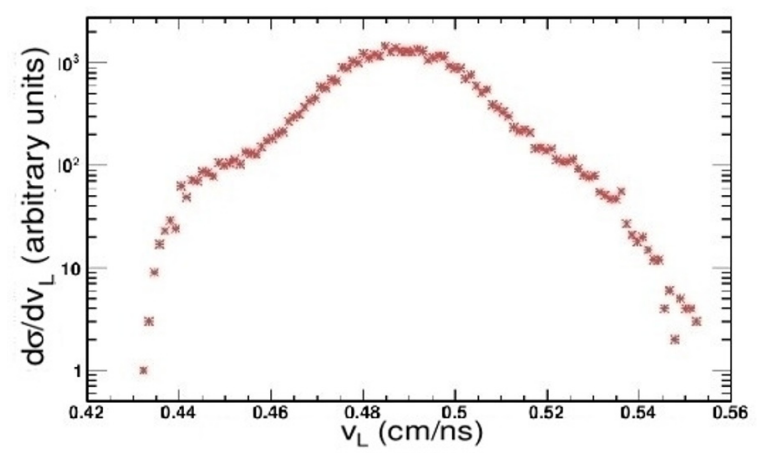

Fig. 7. Measured ER velocity distribution in the laboratory frame for ${ }^{34} \mathrm{~S}+{ }^{89} \mathrm{Y}$ at a beam energy of $124 \mathrm{MeV}$.

\section{Comparison of extracted angular distributions}

Angular distributions of ERs from the $124 \mathrm{MeV}{ }^{34} \mathrm{~S}+{ }^{89} \mathrm{Y}$ reaction are shown in figure 8 , determined using the different techniques discussed in section 3 and 4 . The red points indicate the direct measurement of the angular distribution by scanning in angle using a velocity filter. No transformation techniques are required in this case, hence these data should be considered as the reference. These measurements are described in [8] and a description of the velocity filter method can be found in reference [9].

The angular distribution obtained by transforming the velocity distribution shows fluctuations as noted above, but the overall shape shows quite good agreement with the direct measurement down to $10^{-2}$ of the peak yield at $0^{\circ}$. The angular distribution obtained from the radial distribution on the first MWPC detector shows reasonably good agreement at the larger angles, but generates a narrow peak at the most forward angles which is not present in the direct measurement. The radial distribution in the second MWPC is physically smoothed by angular scattering in the foils of the first MWPC, and can be well-fitted by a single Gaussian form for the angular distribution from the target. As seen in figure 8 this does not reproduce the dip in yield at $5-6^{\circ}$, but overall describes the shape quite well.

The radial distribution method is clearly sensitive to the ER trajectories. In the extreme case, where the focal point is on the MWPC detector, the radial distribution would be primarily determined by two parameters: (i) ER interactions with the gas and (ii) the possible distribution of ER magnetic rigidities resulting from a distribution of ER species which arises from the stochastic nature of the evaporation process. Thus in this case essentially no information on the angular distribution can be obtained. Although the average focal point is determined in the measurement, if the MWPC were too close to the focus, as may have been the case for the first MWPC, the angular distribution could be more sensitive to the two effects described above. In the present results, if these effects increased the ER yield seen in the MWPC close to the axis, the result would be excess yield in the deduced angular distribution close to a scattering angle of $0^{\circ}$.

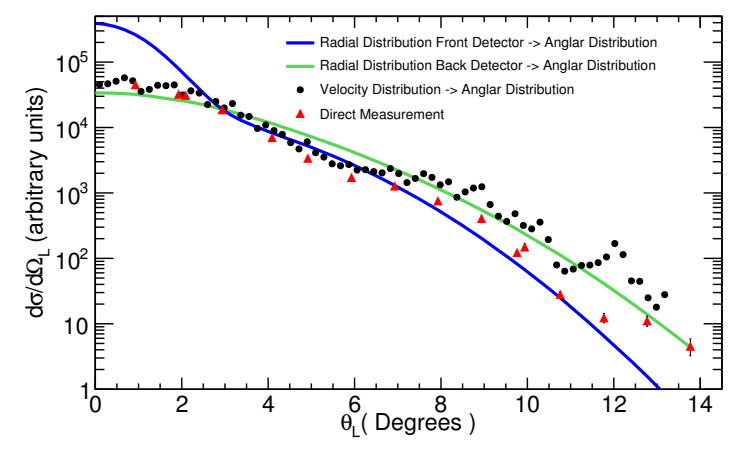

Fig. 8. Comparison of a directly measured angular distribution [8] (red points) with the distributions extracted from the velocity distribution method and from the radial distribution method for the reaction of ${ }^{34} \mathrm{~S}+{ }^{89} \mathrm{Y}$ at a beam energy of $124 \mathrm{MeV}$.

\section{Conclusions}

Based on the results presented, the velocity distribution is the most reliable information for extracting the angular distribution of the ERs exiting the target. Unlike the radial distribution method, it is not sensitive to the position of the focal point that the super-conducting magnet brings the ERs to, nor to details of the interactions of the ERs with the He gas. The effect on the measured velocity distribution of the small losses of ERs through SOLITAIRE, and small deviations from isotropic emission in the centre of mass frame therefore needs to be investigated prior to full application of this method.

\section{References}

1. M. Dasgupta et al., Annu. Rev. Nucl. Part. Sci. 48, (1998) 401

2. M. Dasgupta et al., Phys. Rev. Lett. 99, (2007) 192701

3. M.D. Rodriguez et al., Nucl. Instr. and Meth. A614, (2010) 119

4. R. Rafiei et al., Nucl. Instr and Meth. in Phys. Res. A631 (2011) 12-21

5. A.J. Horsley et al., Nucl. Instr and Meth. A646 (2011) $174-183$

6. M.L. Brown, ANU M.Sc. thesis (2012) unpublished.

7. D.J. Hinde et al., these proceedings

8. A. Mukherjee et al., Phys. Rev. C. 66 (2002) 034607

9. J.X. Wei et al., Nucl. Instr and Meth. A306, (1991) 557562 